\title{
The Moral Dimensions of Open
}

\author{
Karina Ansolabehere, Cheryl Ball, Medha Devare, Tee Guidotti, Bill Priedhorsky, Wim van der Stelt, \\ Mike Taylor, Susan Veldsman, John Willinsky
}

\begin{abstract}
Scholarly publishing is currently undergoing a digital-era transition that provides both opportunities and challenges for improving the moral dimensions of this enterprise. The stakeholders in scholarly publishing need to consider the moral foundations of knowledge production and access that underlie models of scholarly publishing. This report identifies seven moral dimensions and principles to open-access scholarship and data.
\end{abstract}

\section{OSI2016 Workgroup Question}

Does society have a moral imperative to share knowledge freely, immediately, and without copyright restriction? A legal imperative? Why or why not? What about research funded by governments? Corporations? Cancer research? For that matter, is our current mechanism for funding scholarly publishing just or unjust? What other models are there? What are the pros and cons of these models? What is the likelihood of change?

\section{Introduction}

Scholarly publishing is currently undergoing a transition in the digital era that provides both opportunities and challenges for improving the moral dimensions of this enterprise. The stakeholders in scholarly publishing-who range from the inner circles of editors, researchers, publishers, and librarians to the outer reaches of a global and interested public - need to consider the moral foundations of knowledge production and access that underlie models of scholarly publishing.

The moral transition in publishing is the transition between a current paradigm and a new paradigm yet to be fully forged. The current paradigm values classical "liberal" values of ownership of intellectual property, the right to exploit assets that one has legitimately acquired for exclusive personal gain, noninterference with one's own pursuit of knowledge and reward, and additionally, respect for authority, which in publishing comes from the opinions of peers and from vetting via the peer-review process. The new, evolving paradigm stresses freedom of access without impediment or cost (in effect, conferring on knowledge what has previously been a human right), the right of others to access one's own work in detail for their own use in the name of the greater good, an implied commitment to make data freely available notwithstanding the cost and effort to obtain or derive it, surrender of control and possibly credit for the work 
performed as the data and findings are freely disseminated, and abolition or at least dramatic reduction of the gatekeeper role of reviewing, with possible substitution by post-publication peer review.

The legal, if not the moral, case for the current paradigm was forged in the nineteenth century, in an era when scholars were drawn from the elite and privileged spectrum of society; this case seems anachronistic today. At the time, however, it liberated and extended legal protection to scholars and protected the viability of the publishing enterprise on which they depended for dissemination of their work. The new paradigm, which remains to be developed in detail, is well suited for a world in which knowledge is no longer a commodity but a resource that, like water, is necessary for life, and dissemination is no longer a technical problem.

Transition to a new moral paradigm requires that Open be clearly defined and the terms under discussion limited (e.g., post-publication review is not necessarily integral to open access (OA) but is favored by it) so that the implications of each step can be discussed. Everyone's interest is best served by making this discussion explicit, frank, and centered on best use of knowledge. The discussion should be inclusive of all parties and while grounded on utilitarian philosophy, which in this case centers on promoting scholarship and encouraging application over individual gain, must be respectful of the consequences for authors and publishers. The transition will produce losers and winners, each of whom have legal and ethical rights as well as interests at stake.

In this transition period, we need to encourage a period of exploration and grace in the search for new models, while being prepared to judge such efforts by the highest moral standards. We must consider, for example, whether a particular invention maximizes the new digital affordances in order to increase universal access.

We consider it our responsibility to make judgements about the morality of acts, artifacts, systems, and processes, but not on the morality of people and organizations. Judging the latter is dangerous and misleading: nobody judged immoral is beyond redemption, and no one judged moral is beyond error.

Our goal in articulating the moral dimensions of open is to help others, as well as ourselves, understand how to increase the moral and ethical value of our activities and actions in the area of scholarly communication. We see the careful and deliberative articulation of these dimensions to be agents of change in themselves.

\section{Principles/Dimensions}

(1) Most fundamentally, we recognize the moral responsibility to maximize the benefits of scholarly publishing for the larger society. Thus, we need to optimize the dissemination and distribution of scholarly knowledge, for all kinds of users with diverse reading needs, and limit extended embargoes (e.g., they should be no more than 12 months) that prevent researchers, locally and globally, from using, and benefitting from, the most upto-date research.

We want to take full advantage of the introduction of the Internet as a publishing medium without turning our back on the value associated with the historical 
continuities of scholarly publishing. At the forefront of what has changed: duplication of digital information is a mechanism for creating additional value without additional effort. This fundamental change implies a corresponding, foundational moral imperative to take advantage of the opportunity - to extend the value of research as a public good by increasing public access to it.

Because of the huge potential of research to improve the human condition, failure to seek ways of improving scholarship, scientific understanding, and innovation by openly sharing new knowledge and enabling verification of prior results is a moral lapse. Change should be guided by the goal of improving the quality and availability of research and scholarship. This involves reducing barriers to participation that result from economic and geopolitical factors that may impede research contributions, collaborations, and utilization.

(2) The moral argument for open access to scholarly publications is strengthened by the nature of $\mathrm{OA}$ as an enterprise, which is distinguished from other types of publishing, and intellectual property more generally, by several factors:

a) $\mathrm{OA}$ funding models, for example, are based on researchers being paid in advance to conduct research, which includes writing up the work and seeing it published, while not being dependent on royalties from this publication to make a living or being paid for this writing as a work-for-hire, which apply to writers and authors more generally. b) A work of research and scholarship finds its value in its use by others rather than in the extent of its sales. Anything that interferes with or diminishes that use reduces its value to its creator.

c) Claims of veracity or accuracy in a work of research and scholarship depend on the principle of the work being subject to review by any and all; this again calls for the maximum possible access at the time of publication.

(3) Researchers have a moral obligation to make scholarship discoverable, accessible, interoperable, and reusable. This requires budgeting accordingly. Likewise, users of research outputs, including data, have a moral obligation to cite or otherwise give credit to this use in their published research.

(4) Economic models have moral implications (e.g., APCs, subscriptions, "gratis OA," "libre OA," etc.). They should be designed with a view to global fairness as well as how they reward ethical and effective behavior.

(5) Transparency in finance and cost is a moral principle (e.g., where and for what are the costs of publishing and services such as copy-editing, etc.). The moral principle behind cost effectiveness ensures that money is not needlessly spent on publishing that could be spent on research itself, as the economist Ted Bergstrom and colleagues have effectively shown. ${ }^{1}$ Publishers of all varieties should comply with standards of transparency (as far as the law allows) that enable judgement to be made regarding cost effectiveness. Fairness in cost effectiveness is key. In this OA transition, we need transparency of costs and cost effective- 
ness so that all research stakeholders know what publishing costs, why they are paying for publishing services, and how they can budget money towards research, data management, and publishing where appropriate. $^{2}$

Transparency is also relevant to research methods and processes, including peer review, that result in more ethical research and less duplication of results.

(6) The scholarly system has a moral obligation to realign academic incentives. The incentive system must take into account many factors other than the traditional "articles published in prestigious, high impact journals," since the strong emphasis on this measure alone encourages bad behavior such as claim-jumping, inflation or even falsification of results, and over-hyping of the implications of research.

For example, a researcher whose data-set is reused in someone else's subsequent publication deserves credit for their original data contribution. Standards are beginning to be developed for such practices. ${ }^{3}$ We believe that data-sharing must be rewarded if we want this moral, beneficial behavior to increase. At the same time, we believe that researchers and scholarly publishers need to respect rights of privacy, both individual and sociocultural (e.g., indigenous peoples who have a right to their own property and protocols).
(7) Moral ends should be pursued via moral means. This is a broad requirement that can be interpreted in many ways, but may include the following:

- Respect for ethical discourse: Treating each other well and attentively, even when we disagree (some workgroup members conceded that they have not always done well at this).

- Thoughtfulness: Making an effort to avoid, be on the look-out for, and be prepared to remedy the unintended consequences of changes intended to increase the openness of research and scholarship.

- Respect for the rule of law within the academic community, including, but not limited to, respect for intellectual property law. This does not preclude efforts to challenge in the courts or change through legislation the law when it is seen to fail "to promote the progress of Science and the useful Arts" (in the case of the U.S. constitutional clause on intellectual property). Examples include excessive copyright terms barring access to historic publications (which has been challenged unsuccessfully to date in the courts) and rights for researchers to conduct text-mining. The moral underpinning here is that the law is intended to be a reflection of our morality, and that laws change as our moral understanding changes. 


\section{OSI2016 What are the Moral Dimensions of Open? Workgroup}

Karina Ansolabehere, Human rights and democracy expert, FLACSO-Mexico

Cheryl Ball, Director, Digital Publishing Institute, West Virginia University

Medha Devare, Data and Knowledge Manager, Consultative Group on International Agricultural Research (CGIAR)

Tee Guidotti, President-Elect, Sigma Xi

Bill Priedhorsky, Science Resource Office Director, Los Alamos National Laboratory

Wim van der Stelt, Executive Vice President, Projects Open Research, Springer Nature

Mike Taylor, Software Engineer, Index Data and Research Associate, University of Bristol

Susan Veldsman, Director, Scholarly Publishing Unit, Academy of Science of South Africa (ASSAf)

John Willinsky, Professor and Founder of Public Knowledge Project, Stanford University

\section{Notes:}

\footnotetext{
${ }^{1}$ Bergstrom, Theodore C., et al., "Evaluating big deal journal bundles," Proceedings of the National Academy of Sciences, 111.26 (2014): 9425-9430, http://dx.doi.org/10.1073/pnas.1403006111; and Bergstrom, Theodore C., "Free labor for costly journals?," The Journal of Economic Perspectives, 15.4 (2001): 183-198, as of June 16, 2016 : http://eprints.cdlib.org/uc/item/5jc0893p

2 See, for example, "Transparency and Openness Promotion (TOP) Guidelines," Center for Open Science, 2015, as of June 14, 2016: https://cos.io/top/

3 Piwowar, Heather A., and Todd J. Vision. "Data reuse and the open data citation advantage." PeerJ 1 (2013): e175., as of June 14, 2016: https://peerj.com/articles/175/
} 